investigations. The further tests undertaken were at the discretion of the investigating clinician. Statistical analysis was performed using SPSS with Fisher's exact test used to compare categorical data. Results 403 patients (277 female, median age 52 years) who met Rome II criteria for D-IBS were followed-up during the study period. Investigations undertaken in this cohort of patients were as follows: faecal pancreatic elastase (313) gastroscopy (208), colonoscopy (188), glucose or lactose hydrogen breath tests (62), CT/MRI scan (40), barium enema (38), SeHCAT (18) and small bowel capsule endoscopy (18). Final diagnoses are shown in Abstract PWE-056 table 1.88 patients $(22 \%)$ were subsequently identified to have an alternative diagnosis than D-IBS with pancreatic insufficiency and coeliac disease being the most common. When comparing diagnostic yields of individual tests, a lactose-hydrogen breath test had the highest positive yield of $28.6 \%(8 / 28)$, which was significantly higher than any other diagnostic test undertaken $(p<0.01)$.

Conclusion This study highlights the frequency of investigations undertaken in patients with D-IBS symptoms. While the majority of patients have negative tests, there remains a significant number of patients in whom underlying pathology is identified accounting for their D-IBS symptoms. Although NICE guidelines advocate testing for coeliac disease, our study also demonstrates the importance of testing for pancreatic insufficiency in patients presenting with D-IBS symptoms.

Competing interests None declared.

\section{PWE-057 BILATERAL REVERSAL OF A VIRTUAL LESION TO HUMAN PHARYNGEAL MOTOR CORTEX BY CARBONATED WATER SWALLOWING}

doi:10.1136/gutjnl-2012-302514d.57

0 Elshukri, ${ }^{*}$ H Mentz, S Hamdy, S Mistry, E Michou. Inflammation Sciences, University of Manchester (part of the Manchester Academic Health Sciences Centre (MAHSC)), Manchester, UK

Introduction Anecdotal clinical evidence supports the use of carbonated liquids in reducing the likelihood of aspiration in dysphagic patients (Bulow et al. Acta Radiol 2003; Sdravou et al. Dysphagia 2011). Repetitive transcranial magnetic stimulation (rTMS) delivered at $1-\mathrm{Hz}$ can induce transient focal suppression of pharyngeal motor cortex (Mistry S, et al. J Physiol 2007). Here we investigated whether swallowing of carbonated water can reverse the inhibitory effects of a virtual lesion to pharyngeal motor cortex, compared to still water and saliva swallowing.

Methods In 14 healthy subjects (7 male, mean age $34 \pm 15$ (SD)) pharyngeal electromyographic responses were recorded using an intraluminal catheter after the application of Transcranial Magnetic Stimulation (TMS) over pharyngeal motor cortex, as a measure of cortico-bulbar excitability. On three randomised visits, subjects were cued by a visual feedback software to perform a total of 40 swallows over 10 min (swallows every $15 \mathrm{~s}$ ) of either carbonated water or still water vs 10 min of saliva swallowing ad libitum (control), before and after an unilateral $1-\mathrm{Hz}$ virtual lesion of the pharyngeal motor cortex. Cortical excitability (presented as mean \pm SEM) was then reassessed bilaterally for $60 \mathrm{~min}$ post-interventions and analysed with repeated measures ANOVA (SPSS V.14).

Results A three-way ANOVA showed a significant interaction of Hemisphere $\times$ Intervention $\times$ Time $[F(1,13)=5.82, p=0.03]$. Compared to saliva swallowing (control), there was significant increase in cortical excitability bilaterally following swallowing of carbonated water (lesioned pharyngeal motor cortex $[\mathrm{F}(1,13)=7.5$, $p=0.017]$, with a maximum increase of $81 \pm 38 \%$ at $30 \mathrm{~min}$ post intervention (Abstract PWE-057 figure 1A); unlesioned $[\mathrm{F}(1,13)=$ $4.75, p=0.04]$, with a maximum of $46 \pm 16 \%$, immediately post intervention, Abstract PWE-057 figure 1B), not seen with still water swallowing.

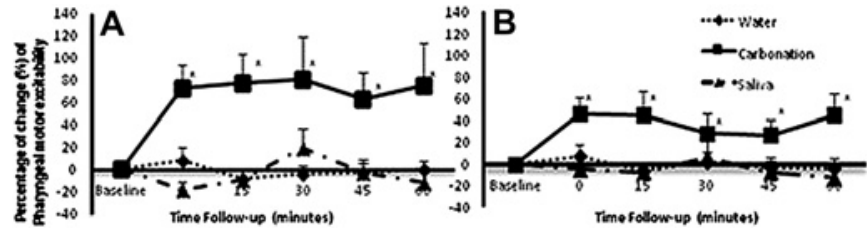

Abstract PWE-057 Figure 1 The effects of carbonation, still water and saliva swallowing to the lesioned $(\mathrm{A})$ and contralateral (non-stimulated pharyngeal motor cortex, B) with $1-\mathrm{Hz}$ fTMS in 14 healthy volunteers, ${ }^{*} p<0.05$.

Conclusion Carbonation reverses the effects of a unilateral virtual lesion with significant increases in cortical excitability not limited to the lesioned hemisphere. These data support the notion that chemesthetic stimuli of carbonation may provide the required peripheral sensory information that can influence brain swallowing activity compared to still water swallowing. These data provides the platform for considering the use of carbonation as facilitating stimuli in dysphagic patients who aspirate thin liquids.

Competing interests None declared.

\begin{tabular}{ll}
\hline PWE-058 & POSTPRANDIAL SUPPRESSION OF REFLUX BY A RAFT \\
FORMING ALGINATE (GAVISCON ADVANCE) COMPARED \\
TO A SIMPLE ANTACID: TECHNICAL ASSESSMENT OF \\
PH-IMPEDANCE MONITORING AND CLINICAL \\
FEASIBILITY STUDY IN GASTRO-OESOPHAGEAL REFLUX \\
DISEASE (GORD) PATIENTS
\end{tabular}

doi:10.1136/gutjnl-2012-302514d.58

${ }^{1} \mathrm{R}$ Sweis, ${ }^{*}{ }^{1} \mathrm{~A}$ Anggiansah, ${ }^{1} \mathrm{~T}$ Wong, ${ }^{2} \mathrm{M}$ R Fox. ${ }^{1}$ Oesophageal Lab, Guys and St Thomas NHS Foundation Trust, London, UK; ${ }^{2}$ NIHR Biomedical Research Unit Nottingham Digestive Diseases Centre, Nottingham, UK

Introduction Proton Pump Inhibitors (PPI) reduce acid reflux but not the frequency or proximal extent of reflux events that are a cause for persistent symptoms on PPI. Alginate preparations containing bicarbonate are effective for short-term control of reflux symptoms by formation of a viscous raft above the meal and acid neutralisation. However mechanistic studies have found equivocal effects of alginates on reflux suppression. It is unclear whether this was due to lack of effect, study power or technical issues.

Aim (i) technical assessment of $\mathrm{pH}$-impedance equipment (Sandhill) in patients taking acid and reflux suppressants (ii) in vivo assessment of mechanistic effects of Gaviscon Advance (GA; Reckitt Benckiser) and Milk of Magnesia (MM; Boots) on postprandial reflux.

Methods (i) To assess effects on signal detection 10 patients took $10 \mathrm{ml}$ GA or MM followed by repeated, single $10 \mathrm{ml}$ swallows of orange juice $(\mathrm{pH} 4)$ until chemical and volume clearance was detected (ii) A randomised, controlled, double-blind, cross-over clinical study in 20 GORD patients (9 male: 11female; age 25-63) referred for investigation of reflux symptoms. On subsequent days at the beginning and end of a $24 \mathrm{~h}$ monitoring period, patients were randomised to receive either $10 \mathrm{ml} \mathrm{GA}$ or $\mathrm{MM}$ (both mint flavoured) after a mixed test meal $(600 \mathrm{kcal})$. Postprandial distal and proximal reflux events (acid and non-acid) were documented over $4 \mathrm{~h}$ by $\mathrm{pH}$ impedance with the patient in the upright, seated position.

Results (i) Technical: After intake of $10 \mathrm{ml} \mathrm{GA}$ or $\mathrm{MM}$ the $\mathrm{pH}$ and impedance signal fully recovered after median $6(2-12)$ and $4(2-10)$ swallows of orange juice. (ii) Clinical: During the $4 \mathrm{~h}$ postprandial observation acid exposure time (mean $2.3 \%$ (SD 3.3\%) vs $3.4 \%$ (4.2\%), $\mathrm{p}=0.296)$ and number of distal reflux events $(20.5$ (13.6) vs 22.5 (9.4), $p=0.500$ ) was similar after ingestion of GA and MM. 
There was a trend to less proximal reflux events with the alginate compared to the antacid (10.5 (8.9) vs 13.9 (8.3), $\mathrm{p}=0.070)$. No difference in the number of symptoms (5.0 (6.0) vs 4.2 (8.3), $\mathrm{p}=0.701)$ and reflux related symptoms (2.5 (4.0) vs 2.8 (5.6), $\mathrm{p}=0.988$ ) was reported.

Conclusion Standard $\mathrm{pH}$-impedance monitoring is suitable for clinical studies of reflux suppression in GORD patients. Distal reflux was similar but a trend to suppression of proximal reflux by GA compared to $\mathrm{MM}$ is present. This feasibility data indicates that trials will require 70 GORD patients to demonstrate effects (power 90\%, p<0.05) on proximal reflux suppression after meals by alginates. More prolonged studies are required to assess effects on symptom control.

Competing interests R Sweis: None declared, A Anggiansah: None declared, $T$ Wong: None declared, M Fox Grant/Research Support from: Reckitt Benckiser.

\section{PWE-059 PREVALENCE OF GASTRO-ESOPHAGEAL REFLUX DISEASE IN INDIVIDUALS WITH IRRITABLE BOWEL SYNDROME: A SYSTEMATIC REVIEW AND META-ANALYSIS}

doi:10.1136/gutjnl-2012-302514d.59

${ }^{1} \mathrm{R}$ M Lovell, ${ }^{* 1,2} \mathrm{~A}$ C Ford. 'Leeds Gastroenterology Institute, Leeds General Infirmary, Leeds, UK; ${ }^{2}$ Leeds Institute of Molecular Medicine, University of Leeds, Leeds, UK

Introduction Irritable bowel syndrome (IBS) and gastro-esophageal reflux disease (GERD) are common disorders in the general population, and there is thought to be a degree of overlap between the two. However, the strength of this association has not been examined systematically. We performed a systematic review and metaanalysis to estimate the prevalence of GERD in individuals with IBS. Methods MEDLINE, EMBASE, and EMBASE Classic were searched through October 2011 for cross-sectional surveys reporting the prevalence of IBS. A recursive search of the bibliographies of identified articles was also conducted. There were no language restrictions. Eligible studies were population-based, recruited $\geq 50$ adult ( $\geq 15$ years) subjects, and were required to define IBS via the Manning or Rome criteria, or according to a questionnaire. Eligibility assessment and data extraction were conducted independently in a double-blind fashion by two investigators, with any discrepancies resolved by consensus. Data were extracted and pooled, with a random effects model, to estimate prevalence of IBS in the population under study. The prevalence of GERD in individuals with and without IBS was compared using an OR, with a 95\% CI. Results The search yielded 20146 results, of which 390 studies appeared relevant and were retrieved for further assessment. There were 80 separate population-based studies that reported the prevalence of IBS according to the various criteria defined above. Of these, 13 studies, containing 49939 participants, also reported the proportion of people who met criteria for GERD within the same population. The pooled prevalence of IBS in these 13 studies was $11.6 \%$ (95\% CI $7.1 \%$ to $17.1 \%$ ). The odds of GERD in individuals with IBS, compared with those without, was 4.17 (95\% CI 2.85 to 6.09). Odds of GERD in individuals with IBS varied according to the criteria used to define IBS (see Abstract PWE-059 table 1).

\section{Abstract PWE-059 Table 1}

\begin{tabular}{lccl}
\hline $\begin{array}{l}\text { Definition of } \\
\text { IBS used }\end{array}$ & $\begin{array}{l}\text { Number of } \\
\text { studies }\end{array}$ & $\begin{array}{l}\text { Number of } \\
\text { subjects }\end{array}$ & OR (95\% CI) \\
\hline Manning & 3 & 5708 & $3.83(1.22$ to 11.97$)$ \\
Rome I & 5 & 6580 & $3.53(2.05$ to 6.10$)$ \\
Rome II & 3 & 18470 & $4.63(2.05$ to 10.44$)$ \\
Rome III & 1 & 18180 & $3.44(2.57$ to 4.59$)$ \\
Questionnaire & 1 & 1001 & $9.59(7.14$ to 12.87$)$ \\
Any definition & 13 & 49939 & $4.17(2.85$ to 6.09$)$ \\
\hline
\end{tabular}

Conclusion The prevalence of GERD in individuals with IBS was fourfold that in those without. The degree of overlap varied according to the criteria used to define IBS, but remained significant in all cases. The strength of this association suggests common pathogenetic mechanisms.

Competing interests None declared.

\section{PWE-060 EFFECT OF GENDER ON PREVALENCE AND SUBTYPE OF IRRITABLE BOWEL SYNDROME: A SYSTEMATIC REVIEW AND META-ANALYSIS}

doi:10.1136/gutjnl-2012-302514d.60

${ }^{1} \mathrm{R}$ M Lovell, ${ }^{* 1,2} \mathrm{~A}$ C Ford. ' ${ }^{1}$ Leeds Gastroenterology Institute, Leeds General Infirmary, Leeds, UK; ${ }^{2}$ Leeds Institute of Molecular Medicine, University of Leeds, Leeds, UK

Introduction It has always been assumed that irritable bowel syndrome (IBS) is more common in women. However, there has been no systematic review and meta-analysis that has synthesised data from all available studies to estimate prevalence of IBS according to gender. There has also been no study that has investigated whether the presumed higher prevalence in women varies according to the way in which IBS is defined, and whether it holds true for all IBS subtypes.

Methods MEDLINE, EMBASE, and EMBASE Classic were searched through October 2011 for cross-sectional surveys reporting the prevalence of IBS. A recursive search was also conducted. There were no language restrictions. Eligible studies were population-based, recruited $\geq 50$ adult ( $\geq 15$ years) subjects, and were required to define IBS via the Manning or Rome I, II or III criteria. Eligibility assessment and data extraction were conducted independently in a double-blind fashion by two investigators, with discrepancies resolved by consensus. Data were extracted and pooled, with a random effects model, to estimate prevalence of IBS according to gender. An OR, with a $95 \% \mathrm{CI}$, was used to compare the female to male prevalence of IBS, as well as the prevalence of constipationpredominant (IBS-C), diarrhoea-predominant (IBS-D), and mixedtype (IBS-M) among women and men meeting criteria for IBS.

Results The search yielded 20146 results, of which 390 studies appeared relevant and were retrieved for further assessment. There were 80 separate population-based studies that reported the prevalence of IBS according to the above criteria. Of these, 55 reported IBS prevalence according to gender, with a pooled prevalence in women of $14.0 \%(95 \%$ CI $11.0 \%$ to $16.0 \%)$ compared with $8.9 \%(95 \% \mathrm{CI}$ $7.3 \%$ to $10.5 \%$ ) in men (OR $1.67 ; 95 \%$ CI 1.53 to 1.82 ). Prevalence was consistently higher in women when all definitions of IBS were considered: the OR for women compared to men was $1.55(95 \% \mathrm{CI}$ 1.35 to 1.78 ) with the Manning criteria, 1.99 (95\% CI 1.76 to 2.25) with Rome I, 1.40 (95\% CI 1.24 to 1.59) with Rome II, and 1.81 (95\% CI 1.36 to 2.39 ) with Rome III. Nine studies, with 63827 participants, also reported the breakdown of IBS according to subtype. Prevalence of IBS-C was significantly higher in women with IBS compared with men (OR 2.38; $95 \%$ CI 1.45 to 3.92 ), IBS-D was less common in women with IBS compared with men (OR 0.45 ; $95 \%$ CI 0.32 to 0.65 ), while prevalence of IBS-M was not significantly different according to gender (OR 1.07; 95\% CI 0.84 to 1.38).

Conclusion Prevalence of IBS was modestly increased in women This observation remained stable according to the various diagnostic criteria used. However, among individuals with IBS, women were more likely to have IBS-C than men, and less likely to have IBS-D. These data suggest that gender may influence IBS subtype.

Competing interests None declared. 